\title{
An alternative derivation of second law results to better relate derivation to practical exergy analysis
}

\author{
Indranil Brahma \\ Mechanical Engineering, \\ Bucknell University, \\ 701 Moore Ave, \\ Lewisburg PA 17837, USA \\ Email: Indranil.brahma@bucknell.edu
}

\begin{abstract}
A more general and physically intuitive alternative to the classical macroscopic derivation of second law results is proposed. Instead of using imaginary reversible processes occurring within heat engines that operate between infinite temperature reservoirs, the new derivation is applicable to any arbitrary control volume across which heat and/or work interactions occur. The arbitrary control volume is discretised into infinitesimally small elements. So-called 'Interface Equations' are developed at the interfaces of these elements, utilising the second law statement that heat transfer occurs from higher to lower temperatures. Terms from the interface equations are then rearranged at each element to show that $d S \geq \frac{d Q}{T}$; all other second-law formulation follow from this result. The derivation allows reversible processes to be mathematically defined, which in turn, allows irreversibilities and entropy generation to be understood in terms of spatial non-uniformity of temperature distribution.
\end{abstract}

Keywords: second law; reversibility; heat engine; Clausius inequality; entropy; entropy generation; exergy.

Reference to this paper should be made as follows: Brahma, I. (2018) 'An alternative derivation of second law results to better relate derivation to practical exergy analysis', Int. J. Exergy, Vol. 25, No. 4, pp.326-338.

Biographical notes: Indranil Brahma is an Associate Professor of Mechanical Engineering at Bucknell University. He earned is $\mathrm{PhD}$ from the University of Wisconsin-Madison in 2005. Prior to working in academia, he worked in the automotive industry for about eight years. His current research focus is physics-based machine learning for complex systems.

This paper is a revised and expanded version of a paper entitled 'Rethinking the macroscopic presentation of the second law of thermodynamics' presented at ASEE Annual Conference, Columbus OH, 25-27 June, 2017.

\section{Introduction}

The approximate sequence of the classical macroscopic presentation of second law concepts and results has not changed for more than a century. All concepts and results 
originate from very specific thought experiments that utilise imaginary reversible processes occurring within heat engines that operate between infinite temperature reservoirs. Figure 1 shows a schematic of the approximate sequence found in engineering textbooks used to teach introductory thermodynamics courses over the last few decades (Cengel and Boles, 2011; Moran et al., 2014; Klein and Nellis, 2012; Borgnakke and Sonntag, 2009; Bejan, 2006; Dugdale, 1996; Wark, 1983; Holman, 1980; Keenan, 1970), based on the edifice constructed by Carnot, Clausius and Kelvin (Kestin and Keenan, 1976). This intellectual journey commencing with the Kelvin-Planck/Clausius statements of the Second Law and culminating with Exergy analysis is mathematically simple but conceptually complex, sometimes spanning more than 200 pages in recent textbooks (Cengel and Boles, 2011). The concepts of exergy, entropy generation and entropy successively follow from the Clausius inequality, all of which are derived from arguments that utilise imaginary reversible heat engines (RHEs) in imaginary situations, e.g., an infinite number of miniature RHEs operating together within a finite cycle (Keenan, 1970). The relationship between work and heat in an RHE in turn, originate from a seemingly arbitrary choice of temperature function used to define the thermodynamic temperature scale (TTS). Ultimately, all second law formulations are derived using reversible processes where properties change uniformly in space over an infinite amount of time. The classical derivation is therefore far removed to any actual problem requiring second-law analysis.

Figure 1 Schematic of the sequence of steps in the classical presentation of the second law in engineering textbooks over the last few decades (Cengel and Boles, 2011; Moran et al., 2014; Klein and Nellis, 2012; Borgnakke and Sonntag, 2009; Bejan, 2006; Dugdale, 1996; Wark, 1983; Holman, 1980; Keenan, 1970). The proposed presentation derives the principle of increase of entropy directly, for any general process involving work and/or heat transfer (see online version for colours)

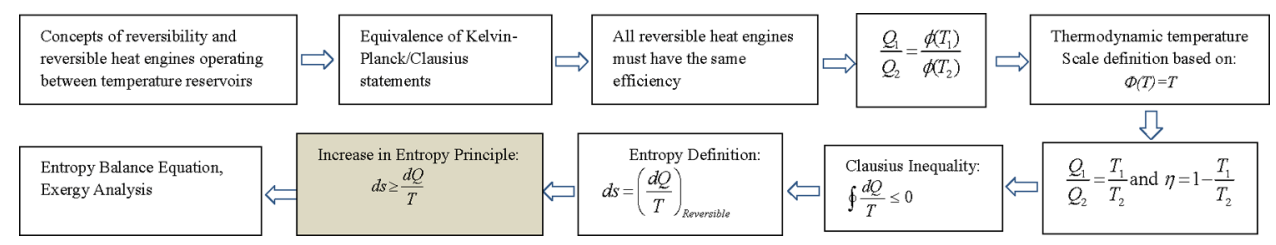

This specific-to-general approach is unusual in science and engineering. It is for this reason that the second law has been expressed and formulated in many different ways for different audiences, e.g., works by Morales (2009), MacDonald (1995), Muschik (1990), Thomsen (1961) and Baierlein (1994). A discussion of different second-law approaches can be found in a review paper by Muschik (1988). Many Introductory physics textbooks at the college level have accordingly modified their presentation of the second law by introducing entropy from a microscopic perspective. However, all of these alternative second law formulations or modified presentations still derive macroscopic results such as the Carnot cycle efficiency or the exergy relations in the same approximate manner as shown in Figure 1. Some introductory physics textbooks (Halliday et al., 1997; Giancoli, 2000; Young et al., 2016; Serway and Jewett, 2014) 
skip the Clausius theorem altogether and address only reversible cycle efficiency starting from $\Delta S=0$. Others derive the Clausius theorem from the Carnot cycle efficiency (Fishbane et al., 1992), which is presented as the upper limit of efficiency (without the RHE corollaries presented in almost all engineering textbooks) and derived for an ideal gas.

The primary distinguishing feature of this work compared to all of the works mentioned above is that the relation $d S \geq \frac{d Q}{T}$ is derived for an arbitrary and real process. As in some of the referenced works, all other results such as the Carnot cycle efficiency and exergy relations follow from $d S \geq \frac{d Q}{T}$.

It is easier to understand the new derivation if a particular example is presented first. This is done in Section 2 followed by the general derivation in Section 3. Conceptual implications are discussed in Section 6.

\section{A particular example}

Consider one-dimensional heat conduction along an insulated rod at any instant of time, as shown in Figure 2. The rod receives an infinitesimal amount of heat $d Q_{\text {source }}$ from an external source and rejects $d Q_{\text {sink }}$ to an external sink during an infinitesimal time period. The rod is not at steady state. It is divided into elements of length $\Delta x$ small enough to ensure uniform properties throughout the element. Since heat transfer is one-dimensional, the temperature decreases monotonically from left to right, as shown in the figure, according to the second law statement that heat transfer occurs from higher to lower temperatures.

Figure 2 One-dimensional unsteady heat conduction along an insulated rod. The rod is divided into infinitesimally small elements. Heat transfer across the boundaries of these elements is used to develop the interface equations. The two terms across each interface shown in the figure are equal in magnitude but different in sign, as indicated by the superscripts. The element (and not the interface) is indicated by the subscript (see online version for colours)

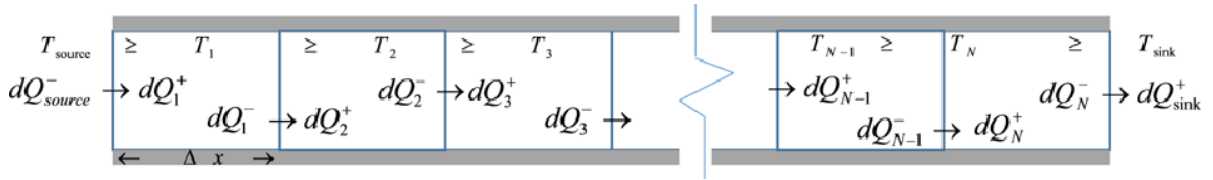

The $d Q$ terms at the interfaces of the infinitesimal elements have a positive superscript if heat is transferred to the element $\left(d Q^{+}\right.$is positive) and a negative superscript if heat is transferred from the element ( $d Q^{-}$is negative) to the next element. The heat transfer across the right interface of element 1 , for example, is $d Q_{1}^{-}$(negative) and is equal to $d Q_{2}^{+}$(positive), the heat transferred to element 2 through it's left interface. Therefore $d Q_{1}^{-}+d Q_{2}^{+}=0$, and since $T_{1}>T_{2}$, it follows that $\frac{d Q_{1}^{-}}{T_{1}}+\frac{d Q_{2}^{+}}{T_{2}}>0$, because the second positive term exceeds the first negative term. Similar equations can be written for all interfaces: 


$$
\begin{array}{lll}
d Q_{\text {source }}^{-}+d Q_{1}^{+}=0 & \text { Since } T_{\text {source }}>T_{1}, & \frac{d Q_{\text {source }}^{-}}{T_{\text {source }}}+\frac{d Q_{1}^{+}}{T_{1}} \geq 0 \\
d Q_{1}^{-}+d Q_{2}^{+}=0 & \text { Since } T_{1}>T_{2}, & \frac{d Q_{1}^{-}}{T_{1}}+\frac{d Q_{2}^{+}}{T_{2}} \geq 0 \\
d Q_{2}^{-}+d Q_{3}^{+}=0 & \text { Since } T_{2}>T_{3}, & \frac{d Q_{2}^{-}}{T_{2}}+\frac{d Q_{3}^{+}}{T_{3}} \geq 0 \\
\ldots \ldots & \\
\ldots \ldots . & \text { Since } T_{N-1}>T_{N}, & \frac{d Q_{N-1}^{-}}{T_{N-1}}+\frac{d Q_{N}^{+}}{T_{N}} \geq 0 \\
d Q_{N-1}^{-}+d Q_{N}^{+}=0 & \text { Since } T_{N}>T_{\text {sink }}, & \frac{d Q_{N}^{-}}{T_{N}}+\frac{d Q_{\text {sink }}^{+}}{T_{\text {sink }}} \geq 0
\end{array}
$$

The equations above will henceforth be referred to as interface equations, since each equation corresponds to an interface. Note that the first and last equations are boundary interfaces. Adding all interface equations:

$$
\frac{d Q_{\text {source }}^{-}}{T_{\text {source }}}+\frac{d Q_{1}^{+}}{T_{1}}+\frac{d Q_{1}^{-}}{T_{1}}+\frac{d Q_{2}^{+}}{T_{2}}+\frac{d Q_{2}^{-}}{T_{2}} \ldots .+\frac{d Q_{N-1}^{-}}{T_{N-1}}+\frac{d Q_{N}^{+}}{T_{N}}+\frac{d Q_{N}^{-}}{T_{N}}+\frac{d Q_{\text {sink }}^{+}}{T_{\text {sink }}} \geq 0
$$

The terms can be re-arranged by collecting them for each node, i.e., the terms can grouped at each element rather than at the interfaces:

$$
\frac{d Q_{\text {source }}^{-}}{T_{\text {source }}}+\left(\frac{d Q_{1}^{+}+d Q_{1}^{-}}{T_{1}}\right)+\left(\frac{d Q_{2}^{+}+d Q_{2}^{-}}{T_{2}}\right) \ldots .+\left(\frac{d Q_{N}^{+}+d Q_{N}^{-}}{T_{N}}\right)+\frac{d Q_{\text {sink }}^{+}}{T_{\text {sink }}} \geq 0
$$

or

$$
\left(\frac{d Q}{T}\right)_{1}+\left(\frac{d Q}{T}\right)_{2} \ldots .+\left(\frac{d Q}{T}\right)_{N} \geq-\left(\frac{d Q_{\text {source }}^{-}}{T_{\text {source }}}+\frac{d Q_{\text {sink }}^{+}}{T_{\text {sink }}}\right)
$$

where $d Q_{i}=d Q_{i}^{+}+d Q_{i}^{-}$is the net heat transfer to any element. Each term on the lefthand-side represents the entropy change. That entropy is a property is shown later in Section 5. Equation (4) can then be written as:

$$
d S_{1}+d S_{2} \ldots .+d S_{N} \geq \frac{d Q_{\text {source }}^{+}}{T_{\text {source }}}+\frac{d Q_{\text {sink }}^{-}}{T_{\text {sink }}}
$$

where $-d Q_{\text {source }}^{-}$and $-d Q_{\text {sink }}^{+}$have been written as $d Q_{\text {source }}^{+}$and $d Q_{\text {sink }}^{-}$respectively. Equation (5) is essentially the famous result $d S \geq \frac{d Q}{T}$, derived without using any reversible processes or heat engines. The Clausius and Carnot theorems, as well as exergy relations, follow readily from it. The general case is derived next. 


\section{The general case}

The example just presented in easy to visualise, but no aspect of the derivation is particular to it. The interface equations (1) hold true for heat transfer at any location, in every situation. The presence or nature of work interactions do not matter, e.g., Figure 2 could represent a thermoelectric element that produces electric work; this would make no difference to the interface equations. Hence interface equations can be written for any arbitrary control volume, and the terms re-grouped to obtain the generalised version of equation (5). The only required modification is a change to generalised notation.

Consider an arbitrary control volume (CV) undergoing work and heat interactions as shown in Figure 3.

Figure 3 The general case of heat transfer occurring across the surfaces of infinitesimal elements (interface equations) within any arbitrary control volume (CV) undergoing arbitrary exchange of work or heat with the environment (see online version for colours)

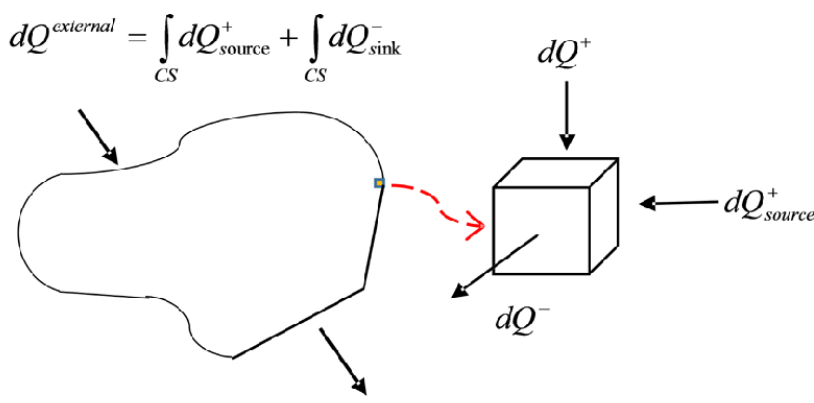

Work

The CV is discretised into infinitesimally small cubic elements, as shown. The interface equations are then true for all surfaces of these three-dimensional elements for the same reason that equation (1) is true, i.e., heat transfer occurs from higher to lower temperature across the interface.

$$
\sum_{\text {All Interfaces }}\left(d Q_{\text {high }}^{-}+d Q_{\text {low }}^{+}\right)=0 \quad \text { Since } T_{\text {high }}>T_{\text {low }}, \quad \sum_{\text {All interfaces }}\left(\frac{d Q_{\text {high }}^{-}}{T_{\text {high }}}+\frac{d Q_{\text {low }}^{+}}{T_{\text {low }}}\right) \geq 0 .
$$

Both terms $d Q_{\text {high }}^{-}$and $d Q_{\text {low }}^{+}$denote the heat transferred across any interface. They are equal in magnitude but opposite in sign. The superscripts denote whether the term is positive or negative, while the subscripts 'high' and 'low' simply refer to the higher and lower temperature sides of the interface. The system is identical to that used for equation (1) and Figure 1.

The analogue of equation (2) is then obtained by separating the summations based on the whether the interface is internal to the $\mathrm{CV}$ or occurs at the surface of the CV, i.e., source or sink interfaces: 


$$
\begin{gathered}
\sum_{\text {Source interfaces }}\left(\frac{d Q_{\text {high }}^{-}}{T_{\text {high }}}+\frac{d Q_{\text {low }}^{+}}{T_{\text {low }}}\right)+\sum_{\text {Internal interfaces }}\left(\frac{d Q_{\text {high }}^{-}}{T_{\text {high }}}+\frac{d Q_{\text {low }}^{+}}{T_{\text {low }}}\right) \\
+\sum_{\text {Sink interfaces }}\left(\frac{d Q_{\text {high }}^{-}}{T_{\text {high }}}+\frac{d Q_{\text {low }}^{+}}{T_{\text {low }}}\right) \geq 0 .
\end{gathered}
$$

If the terms are re-arranged by re-grouping terms within each element rather than at each interface, the analogue of equation (3) results:

$$
\sum_{\text {Source interfaces }} \frac{d Q_{\text {high }}^{-}}{T_{\text {high }}}+\sum_{\text {Internal Elements }}\left(\frac{d Q_{\text {high }}^{-}+d Q_{\text {low }}^{+}}{T}\right)+\sum_{\text {Sink interfaces }} \frac{d Q_{\text {low }}^{+}}{T_{\text {low }}} \geq 0 .
$$

The only interface terms that remain in equation (7) are those that are external to the CV. Noting that $-d Q_{\text {high }}^{-}=d Q_{\text {high }}^{+}$and $-d Q_{\text {low }}^{+}=d Q_{\text {low }}^{-}$, equation (7) can be re-arranged to obtain the analogue of equation (4):

$$
\sum_{\text {Internal Elements }}\left(\frac{d Q_{\text {high }}^{-}+d Q_{\text {low }}^{+}}{T}\right) \geq \sum_{\text {Source Interfaces }} \frac{d Q_{\text {high }}^{+}}{T_{\text {high }}}+\sum_{\text {Sink Interfaces }} \frac{d Q_{\text {low }}^{-}}{T_{\text {low }}}
$$

or

$$
\sum_{\text {Internal Elements }} d S \geq \sum_{\text {Surface Interfaces }} \frac{d Q}{T} .
$$

Note that the positive $d Q^{+}$term and negative $d Q^{-}$terms have been replaced by $d Q$ on the RHS, by following the usual sign convention of heat transfer into the CV being positive and heat loss from the CV being negative. Since the elements need to be small enough for properties to be uniform, they must be infinitesimally small in general. The summations can then be replaced by integration:

$$
\int_{\mathrm{CV}} d S \geq \int_{\mathrm{CS}}\left(\frac{d Q}{T}\right)
$$

The LHS is an integration over infinitesimal elements inside the CV while the RHS is an integration over the control surface of the $\mathrm{CV}$, denoted by $\mathrm{CS}$. Note that the infinitesimal elements change volume during each infinitesimal time step, so that no mass transfer occurs from one element to another. Equation (10) is the main result of this work. All other second law results follow from this, as shown in the next section.

\section{Carnot cycle efficiency and exergy relations}

Both sides of equation (10) are still differential quantities over an infinitesimal time duration, identical to the $d S \geq \frac{d Q}{T}$ result in engineering thermodynamics textbooks, which always consider a finite control volume undergoing quasi-static processes (so there is no reason to integrate over space). Stated in another way, if $q^{\prime \prime}$ is the heat flux, then $d Q=q^{\prime \prime} . A . d t$ in the classical approach but $d Q=q^{\prime \prime} . d A . d t$ in the proposed approach. 
Notice the product of two differentials for time and surface area in the latter term; this necessitates double integration to produce a finite quantity.

If both sides of equation (10) are integrated over time, we obtain:

$$
\Delta S \geq \int_{t} \int_{\mathrm{CS}}\left(\frac{d Q}{T}\right) .
$$

This is the mathematical form of the second law in the proposed approach, i.e., the increase in entropy principle. For any cyclic process, the property change $\Delta S=0$ and equation (11) reduces to the Clausius Inequality:

$$
\oint_{t} \int_{\mathrm{CS}}\left(\frac{d Q}{T}\right) \leq 0 .
$$

It is evident that in order to convert heat into work, at least one heat sink would be required in order for the left-hand-side to be negative. This is essentially the KelvinPlanck statements of the second law. For the limiting case where the CV encloses a cyclic and RHE operating between a single source and a single sink of constant temperature, equation (12) reduces to:

$$
\frac{Q^{\text {Source }}}{T^{\text {Source }}}+\frac{Q^{\text {Sink }}}{T^{\text {Sink }}}=0 .
$$

This results in the familiar expression for the thermal efficiency of a RHE operating between two temperature reservoirs:

$$
\eta_{\text {reversible }}=\frac{Q^{\text {Source }}+Q^{\text {Sink }}}{Q^{\text {Source }}}=1-\frac{T^{\text {Source }}}{T^{\text {Sink }}} .
$$

The second law equation (11) can also be used to determine the exergy of a substance. If a $\mathrm{CV}$ is drawn around any substance to determine its exergy, and the boundaries are drawn far enough so that the heat interactions occur at ambient temperature $T_{o}$ (without a heat source), equation (11) integrates to:

$$
\Delta S \geq \frac{Q}{T_{o}},
$$

where $\Delta S$ corresponds to the change between current and dead state. Maximum work production will correspond to the limiting equality corresponding to an imaginary reversible process:

$$
\Delta Q_{\text {reversible }}=T_{0} \Delta S .
$$

The mathematical expressions for exergy of any closed or open system readily follow from equation (16) when combined with the first law, and can be found in most textbooks. The $\Delta S$ term must include the entropy change of the flow terms if mass crosses the $\mathrm{CV}$ boundaries. 


\section{Entropy as a property}

The interface equations hold true irrespective of the temperature scale used. To establish entropy as a property however, it is necessary to use the ideal gas temperature scale (IGTS). The IGTS coincides with the TTS, but is based on the constant volume thermometer, commonly described in textbooks. Using the IGTS, it is obvious that entropy change for an ideal gas is a property, because of the exact differential:

$$
d s=\frac{d u+p d v}{T}=\left(\frac{C_{v}}{T}\right) d T+\left(\frac{R}{v}\right) d v .
$$

It is easy to extend the argument to non-ideal gas substances by imagining an ideal and non-ideal substance enclosed in infinitesimally small fixed volumes, within a finite $\mathrm{CV}$, undergoing a slow cycle of heating and cooling. The mass of the ideal gas is adjusted so that both infinitesimal volumes have identical heat capacity. The process is slow enough and the packets are small/close enough to result in identical temperature trajectories, as well as identical surrounding temperature trajectories for both infinitesimal volumes. Heat flux between the surroundings and both infinitesimal volumes will therefore be identical. If $\oint \frac{d Q}{T}=0$ for the ideal gas, it must be so for the other arbitrary substance too, since both the numerator and denominator are identical. Hence $\oint \frac{d Q}{T}=0$ for all substances and entropy must be a property. This is a fascinating result because the knowledge of ideal gas behaviour allows us to define a property for any arbitrary substance without any knowledge of the physical processes governing changes in internal energy and work production within that substance.

An alternate method of establishing entropy as a property is to imagine two RHE's operating with an ideal gas and arbitrary non-ideal gas substance respectively. Both must have the same efficiency given by equation (14) so $\Delta S=0$ for the arbitrary substance undergoing a cyclical process.

\section{Comparison with classical approach}

The main difference is in the derivation of $d S \geq \frac{d Q}{T}$. The classical presentation relies on RHE's to derive this result, see Figure 1, and this can be done in a few ways. One commonly used method, e.g., Borgnakke and Sonntag (2009), is to first establish the efficiency of a RHE by doing a thought experiment to show that RHE efficiency can only depend on the ratio of sink and source temperature functions. This temperature function is then chosen to be simply $\Phi(T)=T$ to define the thermodynamics temperature scale (TTS), and to obtain equation (14).

The Clausius inequality $\oint \frac{d Q}{T} \leq 0$ is then obtained with a thought experiment by comparing RHE and non-RHE efficiency by subtracting equation (14) from its nonreversible counterpart (inequality). The Second Law corollary that RHE efficiency cannot be exceeded by any heat engine is employed.

Next, entropy is defined as a property by considering the special reversible case $\oint_{\operatorname{Rev}} \frac{d Q}{T}=0$ and performing a thought experiment involving two cyclical processes that transition from state A to B along different reversible paths, but return through the same 
path, see mid-path in Figure 4 (left). The $\left(\frac{d Q}{T}\right)_{\text {Rev }}$ of the different paths from A to B must, therefore, be identical, since the sum is zero. Therefore $\left(\frac{d Q}{T}\right)_{\text {Rev }}$ must be path-independent and must be a property, termed entropy.

Figure 4 Commonly used thought experiments in the classical presentation to establish entropy as a property (left) and to show that $d S \geq \frac{d Q}{T}$ (right) (see online version for colours)

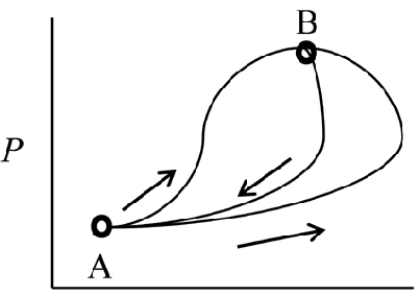

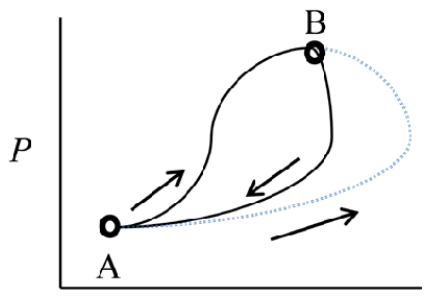

This is then followed by another similar thought experiment, but this time one of the initial paths is irreversible, shown by the dotted line in Figure 4 (right). The Clausius inequality is then applied to both the reversible (equality) and irreversible cycle (inequality). Two equations result, which are then subtracted from each other to obtain $d S \geq \frac{d Q}{T}$, see textbook by Borgnakke and Sonntag (2009) for details.

All exergy relations follow from this result, in a manner sometimes similar to Section 4. Note that the above is one of a few different approaches in the classical presentation, all of which use specific imaginary processes.

The classical presentation is perhaps simpler mathematically, but involves a lengthy chain of reasoning. The proposed derivation might appear to be more complex mathematically, but has a number of conceptual advantages. The conceptual implications of both approaches have been discussed below.

\subsection{Dependence on reversible processes and RHE'S}

The thought experiments of the classical approach depend on imaginary reversible processes and RHE's, making it difficult to relate the derivation to any practical problem. In most of science and engineering, derivations are made for a general case, and then applied to specific problems. This is flipped in the classical approach where the derivations are made using imaginary processes and specific devices, i.e., RHE's and then applied to everything that is not imaginary, and not an RHE.

In contrast, the proposed approach only utilises real processes. Although the mathematics might appear complicated, they only involve two conceptual steps: writing down the interface equations, and re-grouping the terms at each infinitesimal element. The interface equations follow from the simple second-law statement that heat transfer occurs from higher to lower temperature. The use of infinitesimal elements and regrouping of terms is consistent with computational methods used to analyse most contemporary problems. The understanding gained from the derivation is then transferable to practical problems. 
Consider the particular example of Figure 2. If the rod is a thermoelectric element, and at steady state, then the maximum theoretical efficiency is given by equation (14). This is easily understood from equation (5); the LHS will be zero at steady state. But it is conceptually difficult to relate the thought experiments of the classical approach to a thermoelectric element. It is not immediately obvious how results derived for a specific situation, i.e., RHE operating between two temperature reservoirs, would be transferable to another specific device, i.e., a thermoelectric element. This specific-to-general approach is an outlier in engineering science.

Consider another slightly different problem of extracting the maximum possible work from the same heated rod with an initial non-uniform temperature distribution, but with no heat source. An engineer tasked with this problem will discretise the rod into small segments and apply exergy relations to each of them. The only way to conceptually relate the classical derivation to this problem would be to imagine a tiny RHE attached to each segment, but this will add little insight to the physics of the problem at hand. On the other hand, the conceptual framework of the interface equations suggest that maximum work will correspond to an infinitely slow cooling to ambient temperature such that the interface equation (6) is an equality, possible only if the temperature gradient is zero everywhere; resulting in the equality of equation (16). It is physically impossible to achieve zero-temperature gradients, particularly if the rod has an initial non-uniform temperature distribution. But the equality suggests that the rod should be cooled as uniformly and as slowly as possible; a valuable insight for the engineer.

\subsection{Mathematical definition of reversible processes}

Although reversible processes form the backbone of engineering thermodynamics process/cycle analysis, the classical presentation does not define them mathematically. The proposed approach provides a simple definition. A reversible process is any process where the interface equations are zero everywhere, at all times, corresponding to the equality in equation (6). This means that temperature distribution must be uniform, and must change uniformly. This is a physical impossibility, but a mathematical limiting case. Contemporary textbooks seldom state that all processes plotted with solid lines are limiting cases.

\subsection{Conceptual understanding of reversible processes}

Non-zero interface equations imply heat transfer from higher to lower temperature somewhere within the $\mathrm{CV}$, leading to a loss of work potential as per equation (14). This is an important idea that can be used to understand reversible thermodynamic cycles better. Most students have trouble understanding why the Carnot cycle is fundamentally more efficient than other cycles (except the Stirling and Ericson cycle), when operating between identical temperature limits. These 'other' cycles, e.g., the Otto cycle, differ from the Carnot cycle in that heat addition and rejection is not isothermal, but is still reversible. This means that the heat source or sink changes temperature continuously to be equal to the temperature of the working fluid at every instant. If this situation is understood to be equivalent to irreversible heat exchange with constant temperature reservoirs, then equation (14) demonstrates how work potential is destroyed in these cycles but not the Carnot cycle. 
The primary conceptual advantage of the proposed approach is the connection between reversibility and spatial uniformity. A reversible process must be spatially uniform by definition. Any kind of frictional dissipation, including turbulent dissipation, is a result of relative motion, and hence spatially non-uniform by definition. External heat transfer in a reversible process, if any, must occur uniformly across the $\mathrm{CV}$, and not just at the boundaries, to prevent internal temperature gradients that would render the interface equations non-zero. Even an adiabatic and frictionless process such as adiabatic expansion would need to occur infinitely slowly so that property gradients (resulting in internal heat transfer) are absent. Spatial uniformity is obviously impossible to achieve in practice because it requires the process to be infinitely slow. But the concept is important when attempting to conserve work potential in real dynamic processes. For example, exergy destruction during combustion within internal combustion engines can be reduced by initiating the combustion reaction as uniformly as possible throughout the combustion chamber. Such strategies can be observed in recent combustion systems, e.g., Homogenous Charge Compression Ignition (HCCI) engines (Epping et al., 2002). It is obvious from these arguments that a frictionless fluid could undergo an irreversible process due to spatially non-uniform processes, e.g., non-uniform heat addition. This distinction is useful to those who conflate 'reversible' with 'frictionless', and don't understand the difference between the two concepts.

In contrast to all of the above, the classical approach defines reversibility quite broadly, and perhaps vaguely; Holman (1980) for example, defines a reversible process as any process that can be hypothetically reversed without violating the second law of Thermodynamics. Reversibility is explained by illustrating different kinds of frictional and thermal irreversibilities, qualitatively, without a uniting mathematical argument. Spatial uniformity is implied but rarely stated explicitly. No connection is made between irreversibility and spatial non-uniformity of processes and properties. RHE thought experiments are used to show that reversible cycles are more efficient than irreversible cycles but no attempt is made to demonstrate, mathematically or otherwise, that a reversible process conserves work potential.

\subsection{Mathematical understanding of entropy generation and irreversibility}

In the proposed approach, entropy generation can simply be understood as the difference between the RHS and LHS of equation (8). This difference will be large if temperature gradients inside the $\mathrm{CV}$ are large, i.e., higher degree of spatial non-uniformity corresponding to larger inequalities in the interface equations and a larger LHS for equation (6).

No such mathematical interpretation is possible in the classical approach, because everything is derived from imaginary reversible processes. Irreversibilities are often calculated using the entropy generation term derived from entropy balance. Even though the entropy balance equation follows from $d S \geq \frac{d Q}{T}$, it does not add any insight about the physical processes inside the $\mathrm{CV}$. The same can be said about the exergy balance equations. 


\subsection{The thermodynamic temperature scale (TTS)}

The proposed approach starts with the Ideal Gas Temperature Scale (IGTS), uses it for the preceding derivations, and then shows that it coincides with the TTS. The TTS follows from equation (13), i.e., for any RHE:

$$
\frac{T^{\text {Source }}}{T^{\text {Sink }}}=\left|\frac{Q^{\text {Source }}}{Q^{\text {Sink }}}\right| \text {. }
$$

In contrast, the classical approach defines the TTS as a scale that satisfies equation (18), based on an arbitrary choice of temperature function within an RHE thought experiment. Since the TTS is vital to the derivation of every result in the classical presentation, this choice is quite important. The apparent randomness of this choice obscures the fact that if a different temperature function were to be chosen e.g., $\Phi(T)=T^{2}$, then two different temperature scales would be required: the TTS to calculate entropy, and the IGTS for the ideal gas law and other physical laws; a clearly untenable situation. Although the two temperature scales have historically originated from two different concepts (Bejan, 2006), the classical definition of the TTS is therefore very much intertwined with the IGTS.

That the TTS is derived from an imaginary RHE, involves an arbitrary choice, and appears to coincide with the IGTS by chance, can cause considerable confusion to the non-expert.

\section{Conclusion}

Since macroscopic states can only be defined at equilibrium, classical engineering thermodynamics requires imaginary quasi-static reversible processes in order to develop second law results. The proposed derivation takes a differential approach to tackle the non-equilibrium of real processes. It uses properties existing at infinitesimally small elements that result from discretising any arbitrary but finite control volume. The result $d S \geq \frac{d Q}{T}$ is then derived by developing 'interface equations' at the interfaces of these elements, adding them over all elements, and rearranging the terms. All other second law results follow in a conventional manner from this result. The derivation is possibly more mathematically complex than the classical thought experiments with RHEs. However, it is conceptually concise. The interface equations directly follow from the second law statement that heat flows from higher to lower temperatures. No thought experiments with specific processes or systems are required, and no arbitrary choices are made. The interface equations allow a mathematical definition of reversibility, and a conceptual understanding of irreversibility/entropy generation that is tied to temperature gradients resulting from spatial non-uniformity of the process. The author has supplemented the classical approach with the proposed derivation by using only a single 52 min lecture period in an introductory thermodynamics course to alternatively derive $d S \geq \frac{d Q}{T}$. 


\section{Funding}

This research received no specific grant from any funding agency in the public, commercial, or not-for-profit sectors.

\section{References}

Baierlein, R. (1994) 'Entropy and the second law: a pedagogical alternative', American Journal of Physics, Vol. 62, No. 1, pp.15-26.

Bejan, A. (2006) Advanced Engineering Thermodynamics, John Wiley \& Sons, Hoboken, NJ, pp.44-221.

Borgnakke, C. and Sonntag, R.E. (2009) Fundamentals of Thermodynamics, 7th ed., John Wiley \& Sons, Hoboken, NJ, pp.238-420.

Cengel, C.A. and Boles, M.A. (2011) Thermodynamics-An Engineering Approach, 7th ed., McGraw-Hill, New York, NY, pp.277-486.

Dugdale, J.S. (1996) Entropy and its Physical Meaning, Taylor and Francis, CRC Press, Boca Raton, FL, pp.27-62.

Epping, K., Aceves, S., Bechtold, R. and Dec, J. (2002) The Potential of HCCI Combustion for High Efficiency and Low Emissions, SAE Technical Paper 2002-01-1923, DOI: 10.4271/200201-1923.

Fishbane, P.M., Gasiorowicz, S. and Thorton, S.T. (1992) Physics for Scientists and Engineers, 2nd ed., Chapter 20, Prentice-Hall, Upper Saddle River, NJ.

Giancoli, D.C. (2000) Physics for Scientists and Engineers, 3rd ed., Chapter 20, Prentice-Hall, Upper Saddle River, NJ.

Halliday, D., Resnick, R. and Walker, J. (1997) Fundamentals of Physics, 5th ed., Chapter 21, John Wiley \& Sons, Hoboken, NJ.

Holman, J.P. (1980) Thermodynamics, McGraw-Hill, New York, NY, pp.197-238.

Keenan, J.H. (1970) Thermodynamics, MIT Press, Cambridge, MA, pp.58-73.

Kestin, J. (Ed.) (1976) The Second Law of Thermodynamics (Collected Papers of Carnot, Clapeyron, Thomson, Clausius, Planck and Gibb), Dowden, Hutchinson and Ross, UK.

Klein, S. and Nellis, G. (2012) Thermodynamics, Cambridge University Press, Cambridge, England, pp.204-384.

MacDonald, A. (1995) 'A new statement of the second law of thermodynamics', American Journal of Physics, Vol. 63, No. 12, pp.1122-1127.

Morales, A. (2009) 'The second law of classical thermodynamics stated in terms of twin systems', American Journal of Physics, Vol. 77, No. 4, pp.365-372.

Moran, M.J., Shapiro, N.H., Boettner, D.D. and Bailey, M.B. (2014) Fundamentals of Engineering Thermodynamics, 8th ed., John Wiley \& Sons, Hoboken, NJ, pp.241-436.

Muschik, W. (1988) 'Formulations of the second law-recent developments', J. Phys. Chem. Solids, Vol. 49, pp.709-720.

Muschik, W. (1990) 'Second law: Sears-Kestin statement and Clausius inequality', American Journal of Physics, Vol. 58, No. 3, pp.241-244.

Serway, R.A. and Jewett, J.W. (2014) Physics for Scientists and Engineers, Vol. 1, 9th ed., Chapter 22, Cengage Learning, Boston, MA.

Thomsen, J.S. (1961) 'Operational formulation of the second law of thermodynamics', American Journal of Physics, Vol. 29, No. 5, pp.300-307.

Wark, K. (1983) Thermodynamics, McGraw-Hill, New York, NY, pp.195-334.

Young, H.D., Freedman, R.A. and Ford, L.A. (2016) University Physics, 14th ed., Chapter 20, Pearson, London, UK. 